\title{
New limits on heavy neutrinos at NA62
}

\author{
Chris Parkinson ${ }^{* \dagger}$ \\ University of Birmingham \\ E-mail: chris.parkinsonecern.ch
}

The NA62 experiment at CERN collected a large sample of charged kaon decays in flight with a minimum bias trigger in 2007. Upper limits on the rate of the charged kaon decay into a muon and a heavy neutrino obtained from this data are reported for a range of heavy neutrino masses. Prospects for similar searches using data collected in 2015 are also reported.

XXV International Workshop on Deep-Inelastic Scattering and Related Subjects 3-7 April 2017

University of Birmingham, $U K$

\footnotetext{
*Speaker.

${ }^{\dagger}$ for the NA62 Collaboration: R. Aliberti, F. Ambrosino, R. Ammendola, B. Angelucci, A. Antonelli, G. Anzivino, R. Arcidiacono, M. Barbanera, A. Biagioni, L. Bician, C. Biino, A. Bizzeti, T. Blazek, B. Bloch-Devaux, V. Bonaiuto, M. Boretto, M. Bragadireanu, D. Britton, F. Brizioli, M.B. Brunetti, D. Bryman, F. Bucci, T. Capussela, A. Ceccucci, P. Cenci, V. Cerny, C. Cerri, B. Checcucci, A. Conovaloff, P. Cooper, E. Cortina Gil, M. Corvino, F. Costantini, A. Cotta Ramusino, D. Coward, G. D’Agostini, J. Dainton, P. Dalpiaz, H. Danielsson, N. De Simone, D. Di Filippo, L. Di Lella, N. Doble, B. Dobrich, F. Duval, V. Duk, J. Engelfried, T. Enik, N. Estrada-Tristan, V. Falaleev, R. Fantechi, V. Fascianelli, L. Federici, S. Fedotov, A. Filippi, M. Fiorini, J. Fry, J. Fu, A. Fucci, L. Fulton, E. Gamberini, L. Gatignon, G. Georgiev, S. Ghinescu, A. Gianoli, M. Giorgi, S. Giudici, F. Gonnella, E. Goudzovski, C. Graham, R. Guida, E. Gushchin, F. Hahn, H. Heath, T. Husek, O. Hutanu, D. Hutchcroft, L. Iacobuzio, E. Iacopini, E. Imbergamo, B. Jenninger, K. Kampf, V. Kekelidze, S. Kholodenko, G. Khoriauli, A. Khotyantsev, A. Kleimenova, A. Korotkova, M. Koval, V. Kozhuharov, Z. Kucerova, Y. Kudenko, J. Kunze, V. Kurochka, V.Kurshetsov, G. Lanfranchi, G. Lamanna, G. Latino, P. Laycock, C. Lazzeroni, M. Lenti, G. Lehmann Miotto, E. Leonardi, P. Lichard, L. Litov, R. Lollini, D. Lomidze, A. Lonardo, P. Lubrano, M. Lupi, N. Lurkin, D. Madigozhin, I. Mannelli, G. Mannocchi, A. Mapelli, F. Marchetto, R. Marchevski, S. Martellotti, P. Massarotti, K. Massri, E. Maurice, M. Medvedeva, A. Mefodev, E. Menichetti, E. Migliore, E. Minucci, M. Mirra, M. Misheva, N. Molokanova, M. Moulson, S. Movchan, M. Napolitano, I. Neri, F. Newson, A. Norton, M. Noy, T. Numao, V. Obraztsov, A. Ostankov, S. Padolski, R. Page, V. Palladino, C. Parkinson, E. Pedreschi, M. Pepe, M. Perrin-Terrin, L. Peruzzo, P. Petrov, F. Petrucci, R. Piandani, M. Piccini, J. Pinzino, I. Polenkevich, L. Pontisso, Yu. Potrebenikov, D. Protopopescu, M. Raggi, A. Romano, P. Rubin, G. Ruggiero, V. Ryjov, A. Salamon, C. Santoni, G. Saracino, F. Sargeni, V. Semenov, A. Sergi, A. Shaikhiev, S. Shkarovskiy, D. Soldi, V. Sougonyaev, M. Sozzi, T. Spadaro, F. Spinella, A. Sturgess, J. Swallow, S. Trilov, P. Valente, B. Velghe, S. Venditti, P. Vicini, R. Volpe, M. Vormstein, H. Wahl, R. Wanke, B. Wrona, O. Yushchenko, M. Zamkovsky, A. Zinchenko.
} 


\section{Introduction}

The experimental observation of neutrino oscillations demands neutrino mass terms be added to the Standard Model (SM). The $v$ MSM does this by introducing heavy neutrino mass states that mix with the SM flavour states, and predicts right-handed neutrinos at the GeV scale [1,2].

A heavy neutrino with mass $m_{\mathrm{h}} \lesssim 380 \mathrm{MeV} / \mathrm{c}^{2}$ is kinematically accessible in the decay of the $K^{+}$to a $\mu^{+}$and a muon neutrino. The branching fraction of the decay involving the heavy neutrino, $B\left(K^{+} \rightarrow \mu^{+} v_{h}\right)$, can be expressed as

$$
B\left(K^{+} \rightarrow \mu^{+} v_{h}\right)=B\left(K^{+} \rightarrow \mu^{+} v\right) \cdot f\left(\mathrm{~m}_{\mathrm{h}}\right) \cdot\left|U_{\mu 4}\right|^{2}
$$

where $B\left(K^{+} \rightarrow \mu^{+} v\right)$ is the branching fraction in the SM, $\left|U_{\mu 4}\right|^{2}$ is the squared mixing element between the muon flavour state and the heavy neutrino mass state labelled ' 4 ', and $f\left(\mathrm{~m}_{\mathrm{h}}\right)$ is a numerical factor that accounts for phase-space and helicity suppression [3].

The $K^{+} \rightarrow \mu^{+} v_{h}$ decay is ideal for heavy neutrino production searches. Decays to heavy neutrinos can be easily distinguished from the SM process by the missing mass: $\mathrm{m}_{\mathrm{N}}^{2}=p_{\mathrm{N}}^{2}=$ $\left(p_{K}-p_{\mu}\right)^{2}$, where $p$ is the four-momentum of a given particle and $\mathrm{N}$ is used to denote either the SM neutrino $v$ or the heavy neutrino $v_{h}$. The SM decay is prolific, given it's branching fraction of $64 \%$, meaning substantial samples can be collected via minimum-bias triggers, even with sizeable downscales. Moreover, the mass of the heavy neutrino acts to lift the strong helicity suppression of the SM decay, meaning that if $\left|U_{\mu 4}\right|=1$ the decay rate of $K^{+} \rightarrow \mu^{+} v_{h}$ can be more than four times larger than that of the SM process $K^{+} \rightarrow \mu^{+} v$ depending on the heavy neutrino mass, see Fig. 1(a). The advantages of searches for heavy neutrino production are two-fold: limits on $\left|U_{\mu 4}\right|^{2}$ scale linearly with the number of $K^{+} \rightarrow \mu^{+} v$ decays observed; and limits on $\left|U_{\mu 4}\right|^{2}$ can be set regardless of whether lepton-number-violating processes are induced by the heavy neutrino.

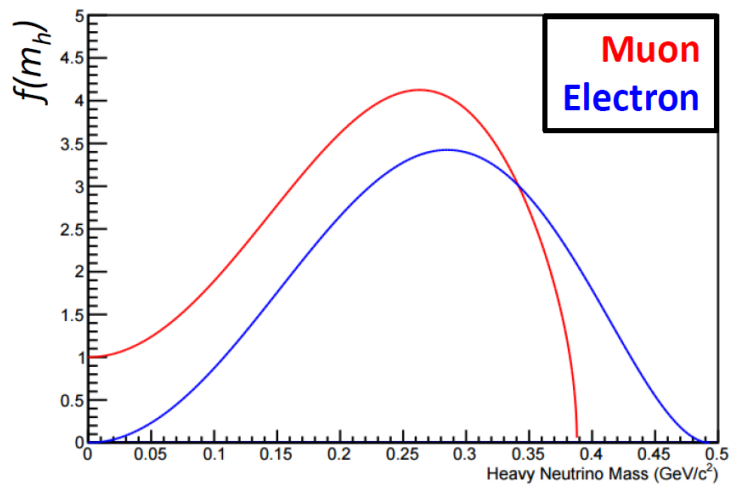

(a)

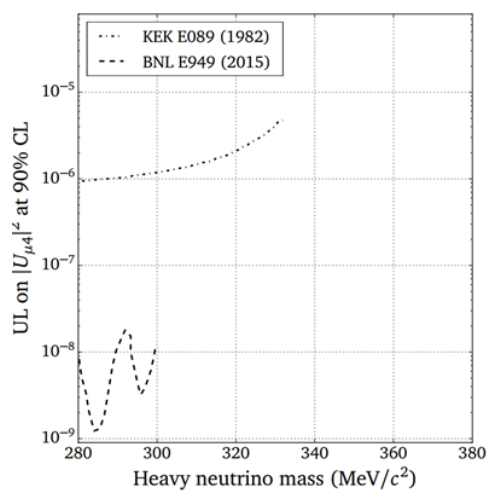

(b)

Figure 1: Value of $f\left(\mathrm{~m}_{\mathrm{h}}\right)$ normalised to the $K^{+} \rightarrow \mu^{+} v$ branching fraction assuming $\left|U_{\mu 4}\right|=1$ for $K^{+} \rightarrow \mu^{+} v_{h}$ decays (red) and $\left|U_{e 4}\right|=1$ for $K^{+} \rightarrow e^{+} v_{h}$ decays (blue) (a), and the current experimental limits on $\left|U_{\mu 4}\right|^{2}$ (b), both as a function of $m_{\mathrm{h}}$.

Existing limits on heavy neutrino production are at the level of $\sim 10^{-8}$ for heavy neutrino masses from 175 to $300 \mathrm{MeV} / c^{2}$ [4] and at the level of $\sim 10^{-6}$ for heavy neutrino masses from 70 to $330 \mathrm{MeV} / \mathrm{c}^{2}$ [5], as shown in Fig. 1(b). A region of interest remains between the kinematic limit, which is around $380 \mathrm{MeV} / c^{2}$, and the existing experimental limits. This region of interest has been 
probed by the NA62 experiment at CERN. The NA62 experimental setup in 2007 and in 2015 is reported in Sec. 2. New limits on heavy neutrino production based on data collected in 2007 are reported in Sec. 3. Prospects for similar measurements based on the data collected by NA62 during 2015 are reported in Sec. 4.

\section{The NA62 experiment at CERN}

The kaon physics programme at NA62 begins with a beam of $p=400 \mathrm{GeV}$ protons extracted from the CERN Super Proton Synchrotron (SPS). The protons are directed onto a Beryllium target, producing secondary hadrons of which about 6 percent are kaons. Those secondary hadrons are formed into beams, with charge(s) and momenta selected using an achromat formed of several dipole magnets. The beams are then focused and transported to the NA62 detector.

\subsection{The NA62 experiment in 2007}

The NA62 experiment utilises both positive and negative beams, which were required to have momenta of $74 \pm 1.4 \mathrm{GeV}$ and could be run together or separately. Periods where only the positive (negative) beam enters the detector are known as $K^{+}\left(K^{-}\right)$periods. Periods where neither beam enters the detector are known as K-less periods. The beams were directed through a $114 \mathrm{~m}$ vacuum tank, which defined the fiducial volume (decay volume) of the experiment. The beam hadrons were accompanied by a flux of stray "halo" muons produced by kaon and pion decays upstream of the decay volume.

In 2007 the NA62 detector was the same as that of the earlier NA48/2 experiment, which is described in detail in Ref. [6] and is shown in Fig. 2. The momenta of charged particles were measured by a magnetic spectrometer that consisted of four drift chambers immersed in Helium, and a dipole magnet. The spectrometer had a momentum resolution of $\sigma_{p} / p=0.48 \% \oplus 0.009 \% \cdot p$, where the momentum $p$ is expressed in $\mathrm{GeV} / c$. A charged-particle hodoscope (CHOD), which consisted of two planes of plastic scintillator strips, was used to produce fast signals for the trigger system. The liquid Krypton (LKr) electromagnetic calorimeter had 13248 readout cells without longitudinal segmentation, providing high granularity in the transverse plane. The LKr energy resolution was $\sigma_{E} / E=3.2 \% / \sqrt{E} \oplus 9 \% / E \oplus 0.42 \%$, where $E$ is expressed in $\mathrm{GeV}$, and the spatial resolution was $\sigma_{x}=\sigma_{y}=0.42 \mathrm{~cm} / \sqrt{\mathrm{E}} \oplus 0.06 \mathrm{~cm}$. A muon-veto (MUV) system composed of three planes of plastic scintillator strips was located at the downstream-end of the detector.

\subsection{The NA62 experiment in 2015}

The 2015 experimental setup is described in detail in Ref. [7]. Aside from beam-line modifications so that only a positive hadron beam could be transported to the detector, a magnet system was introduced that increased the beam intensity by a factor of 90 .

A schematic of the NA62 detector as it was 2015 is shown in Fig. 3. The KTAG and GTK detectors make a precise measurement of the time and momenta of each kaon that enters the decay volume. The drift chambers used in 2007 were replaced by four stations of straw tubes operated in vacuum. A hermetic system of photon vetoes means that $K^{+}$decays that produce a $\pi^{0}$ in the final state can be efficiently removed. The old MUV system was replaced by a single layer of transversely segmented plastic scintillator (MUV3). 


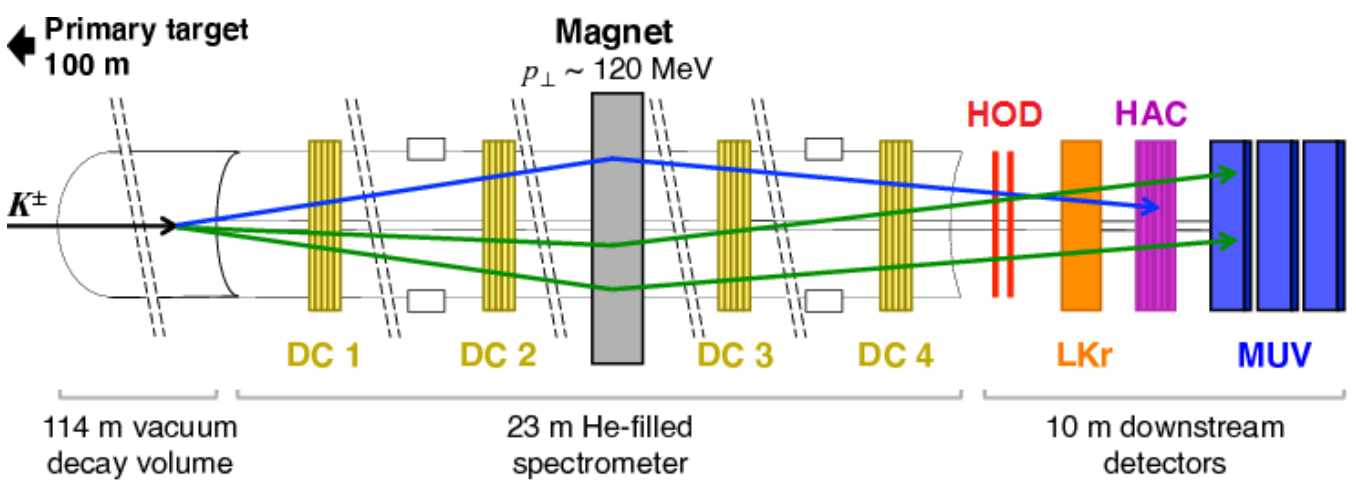

Figure 2: The NA62 detector in 2007.

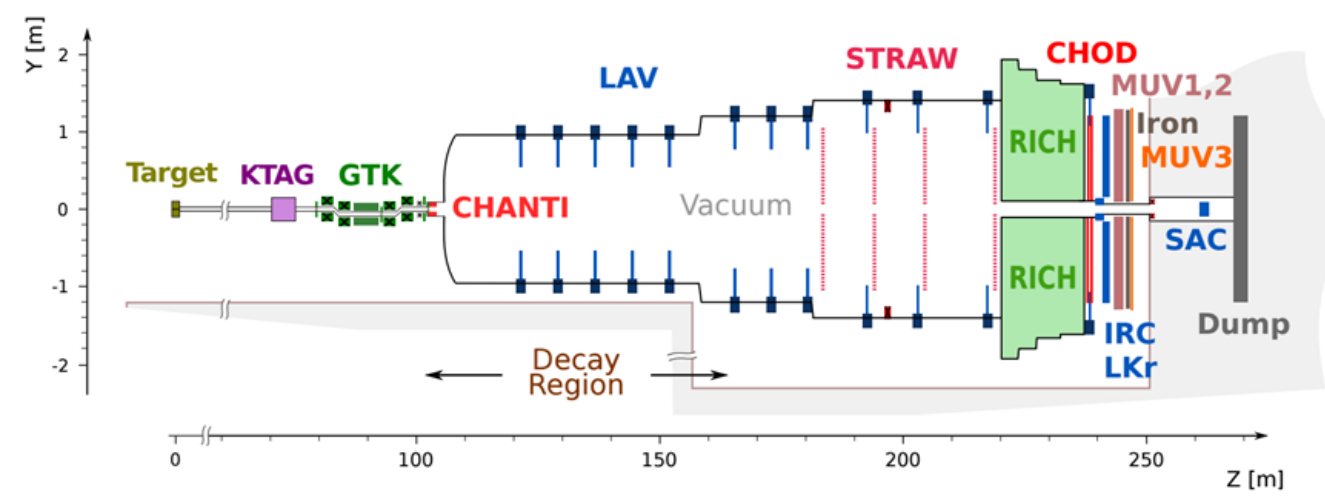

Figure 3: The NA62 detector in 2015.

\section{New limits on heavy neutrinos with the 2007 data}

Events were selected by the trigger system based on the presence of coincident signals in time and space in the two CHOD planes, along with loose upper- and lower- requirements on the hit multiplicity in the drift chambers. A downscaling of 150 was applied at the trigger level. The level of background was found to be substantially lower during the $\mathrm{K}^{+}$periods, so only these periods are used for the measurement. As such, candidate $K^{+} \rightarrow \mu^{+} N$ decays are selected by requiring there to be exactly one positively charged track reconstructed in the spectrometer.

The track is required: to be within the geometrical acceptance of the $\mathrm{LKr}$ and MUV detectors; to have a momentum between 10 and $65 \mathrm{GeV} / c$; to be within $\pm 20 \mathrm{~ns}$ of the trigger time recorded by the CHOD; to have a distance of closest approach (CDA) to the beam axis of less than $3 \mathrm{~cm}$; and to be associated in time and space with signals from the first two planes of the MUV detector. Events are rejected if there are clusters of energy greater than $2 \mathrm{GeV}$ reconstructed in the $\mathrm{LKr}$ that are not consistent with the track. About $8 \mathrm{M}$ candidate $K^{+} \rightarrow \mu^{+} v$ decays are selected in the 2007 data. The missing mass spectrum of candidate $K^{+} \rightarrow \mu^{+} N$ decays is shown in Fig. 4(a). The background is dominated by $K^{+} \rightarrow \pi^{0} \mu \nu$ decays, along with a sizeable contribution from halo muons.

The search for heavy neutrino production is made by setting a limit on the number of $K^{+} \rightarrow \mu^{+} v_{h}$ decays using the Rolke-Lopez method [8]. The limit is computed using the number of observed and expected events in a given search window, along with the uncertainty on the number of expected 
events in that window. The limit is computed for heavy neutrino masses across a search region that extends from 300 to $375 \mathrm{MeV} / c^{2}$ in steps of $1 \mathrm{MeV} / c^{2}$. The size of the search window at each step is defined by the heavy neutrino mass resolution at that step, which reduces from $\sim 5$ to $\sim 1 \mathrm{MeV} / c^{2}$ across the search region. The expected number of events is estimated using simulated events. The uncertainty on the expected number of events is dominated by the halo muon contribution, which is modelled using data collected during $\mathrm{K}^{-}$and $\mathrm{K}$-less running periods.

The computed upper limit on the number of heavy neutrino decays is shown in Fig. 4(b). The limit can be converted to a limit on $\left|U_{\mu 4}\right|^{2}$ using equation 1.1. Limits are set on heavy neutrino production at the level of $\sim 10^{-6}$ for heavy neutrino masses from 300 to $375 \mathrm{MeV} / \mathrm{c}^{2}$, as shown in Fig. 4(c). The limit on $\left|U_{\mu 4}\right|^{2}$ is compared to existing limits on heavy neutrino production in Fig. 4(d). The NA62 limits are more stringent than existing limits in the $325 \lesssim \mathrm{m}_{\mathrm{h}} \lesssim 375 \mathrm{MeV} / \mathrm{c}^{2}$ range.

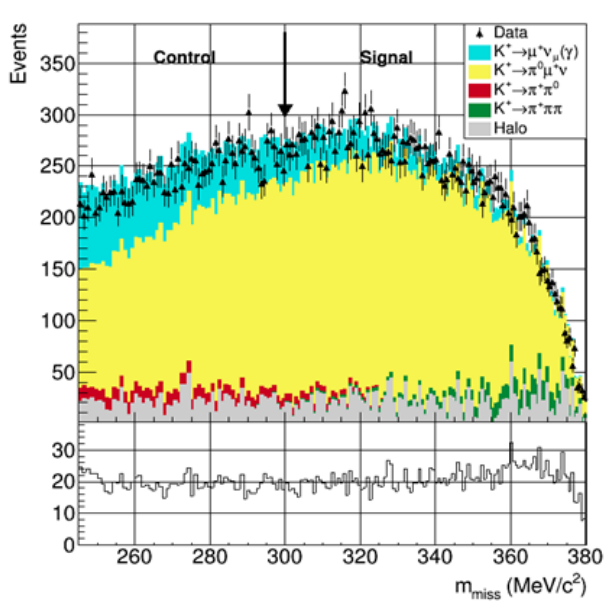

(a)

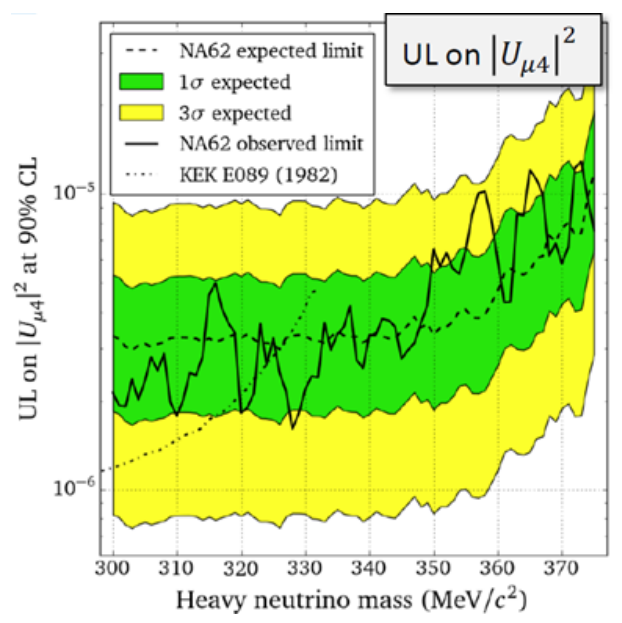

(c)

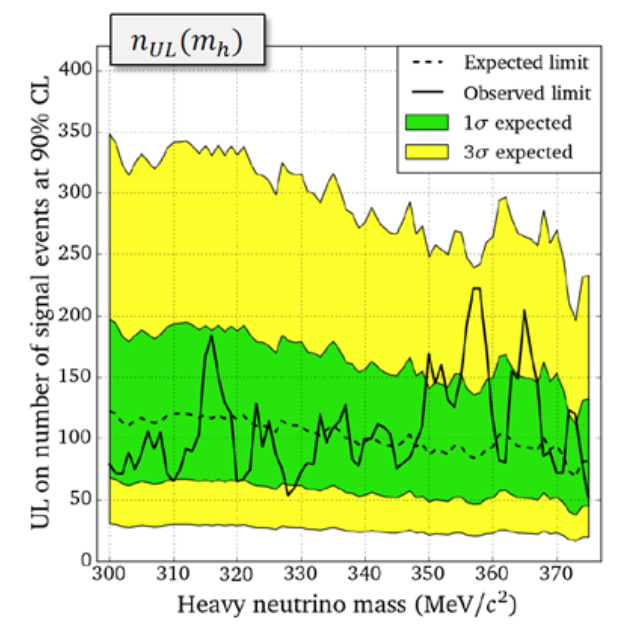

(b)

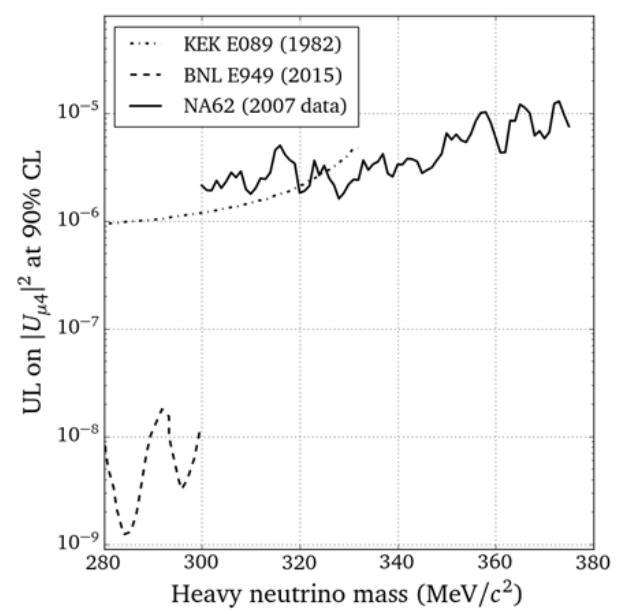

(d)

Figure 4: The distribution of candidate $K^{+} \rightarrow \mu^{+} N$ events in the NA62 data collected in 2007, overlaid with the distribution of simulated events (a). As a function of $\mathrm{m}_{\mathrm{h}}$ : limit on the number of $K^{+} \rightarrow \mu^{+} v_{h}$ decays in the data (b); limit on $\left|U_{\mu 4}\right|^{2}$ (c); comparison of the NA62 limits on $\left|U_{\mu 4}\right|^{2}$ with existing limits (d). 


\section{Prospects for heavy neutrino searches with the 2015 data}

During 2015 the NA62 experiment collected data using a minimum-bias trigger selection over a period of five days. A preliminary analysis of the data shows that around $23 \mathrm{M}$ candidate $K^{+} \rightarrow \mu^{+} v$ decays satisfy the trigger and selection criteria, corresponding to a single event sensitivity $\sim 10^{-8}$. The background level in the heavy neutrino search region is a factor of 100 lower than in 2007.

The lower level of background in 2015 means that, unlike the 2007 data, the 2015 data can also be used to set competitive limits on heavy neutrino production in the $K^{+} \rightarrow e^{+} v$ decay. Limits on the electron mode impose constraints to the $\left|U_{e 4}\right|^{2}$ mixing element, and due to the mass of the electron being smaller than that of the muon, also extends the range of accessible heavy neutrino masses beyond $\sim 380 \mathrm{MeV} / c^{2}$, as seen in Fig. 1(a). A preliminary analysis of the 2015 data shows that about 1500 candidate $K^{+} \rightarrow e^{+} v$ decays satisfy the trigger and selection criteria. Given the low level of background, the analysis of 2015 data from NA62 is expected to improve existing limits on the $K^{+} \rightarrow e^{+} v_{h}$ decay by an order of magnitude.

\section{Summary}

The NA62 experiment is probing an interesting region of heavy neutrino parameter space by searching for heavy neutrino production in the $K^{+} \rightarrow \mu^{+} v_{h}$ decay. Using NA62 data collected in 2007 the world's most stringent limits on heavy neutrino production were set at the level of $\sim 10^{-6}$ for heavy neutrino masses in the range $325 \lesssim \mathrm{m}_{\mathrm{h}} \lesssim 375 \mathrm{MeV} / \mathrm{c}^{2}$. The prospects for improved limits on heavy neutrino production based on the NA62 data collected in 2015 were also reported. Improvements of one order of magnitude beyond existing limits are possible in $K^{+} \rightarrow \mu^{+} v$ and $K^{+} \rightarrow e^{+} v$ decays.

\section{References}

[1] T. Asaka and M. Shaposhnikov, The nuMSM, dark matter and baryon asymmetry of the universe, Phys. Lett. B620 (2005) 17-26.

[2] T. Asaka, S. Blanchet and M. Shaposhnikov, The nuMSM, dark matter and neutrino masses, Phys. Lett. B631 (2005) 151-156.

[3] R. E. Shrock, New Tests For, and Bounds On, Neutrino Masses and Lepton Mixing, Phys. Lett. 96B (1980) 159-164.

[4] E949 Collaboration collaboration, A. V. Artamonov, B. Bassalleck, B. Bhuyan, E. W. Blackmore, D. A. Bryman, S. Chen et al., Search for heavy neutrinos in $K^{+} \rightarrow \mu^{+} v_{H}$ decays, Phys. Rev. D 91 (Mar, 2015) 052001.

[5] R. S. Hayano, T. Taniguchi, T. Yamanaka, T. Tanimori, R. Enomoto, A. Ishibashi et al., Heavy-neutrino search using $K_{\mu 2}$ decay, Phys. Rev. Lett. 49 (Nov, 1982) 1305-1309.

[6] NA48/2 collaboration, J. R. Batley et al., Search for direct CP violating charge asymmetries in $K+-$ $\longrightarrow$ pi+- pi+ pi- and $\mathrm{K+-} \longrightarrow$ pi+- piO piO decays, Eur. Phys. J. C52 (2007) 875-891.

[7] NA62 collaboration, E. Cortina Gil et al., The Beam and detector of the NA62 experiment at CERN, JINST 12 (2017) P05025.

[8] W. A. Rolke, A. M. Lopez and J. Conrad, Limits and confidence intervals in the presence of nuisance parameters, Nucl. Instrum. Meth. A551 (2005) 493-503. 